\title{
Corrigendum
}

\section{Revisiting Latino identity}

Latino Studies (2014) 12, 328. doi:10.1057/lst.2014.34

Correction to: Latino Studies (2014) 12, 4-6. doi: 10.1057/lst.2014.19

In the title of this Editorial, "Revisiting" was incorrectly given as "Revisting." The editor and publishers apologize for this error. 